Article

\title{
Existence, Uniqueness and Exponential Stability of Periodic Solution for Discrete-Time Delayed BAM Neural Networks Based on Coincidence Degree Theory and Graph Theoretic Method
}

\author{
Manickam Iswarya ${ }^{1}$, Ramachandran Raja ${ }^{2} \oplus$, Grienggrai Rajchakit ${ }^{3}(0)$ Jinde Cao ${ }^{4, *}{ }^{\oplus}$, \\ Jehad Alzabut ${ }^{5}$ (D) and Chuangxia Huang ${ }^{6, *(D)}$ \\ 1 Department of Mathematics, Alagappa University, Karaikudi 630 004, India; iswaryajm19@gmail.com \\ 2 Ramanujan Centre for Higher Mathematics, Alagappa University, Karaikudi 630 004, India; \\ rajarchm2012@gmail.com \\ 3 Department of Mathematics, Maejo University, Chiangmai 50290, Thailand; griengkrai@yahoo.com \\ 4 School of Mathematics, Southeast University, Nanjing 211189, China \\ 5 Department of Mathematics and General Sciences, Prince Sultan University, Riyadh 11586, Saudi Arabia; \\ jalzabut@psu.edu.sa \\ 6 Hunan Provincial Key Laboratory of Mathematical Modeling and Analysis in Engineering, \\ Department of Applied Mathematics, Changsha University of Science and Technology, \\ Changsha 410114, China \\ * Correspondence: jdcao@seu.edu.cn (J.C.); cxiahuang@csust.edu.cn (C.H.)
}

Received: 25 August 2019; Accepted: 24 October 2019 ; Published: 4 November 2019

Abstract: In this work, a general class of discrete time bidirectional associative memory (BAM) neural networks (NNs) is investigated. In this model, discrete and continuously distributed time delays are taken into account. By utilizing this novel method, which incorporates the approach of Kirchhoff's matrix tree theorem in graph theory, Continuation theorem in coincidence degree theory and Lyapunov function, we derive a few sufficient conditions to ensure the existence, uniqueness and exponential stability of the periodic solution of the considered model. At the end of this work, we give a numerical simulation that shows the effectiveness of this work.

Keywords: discrete-time BAMNNs; periodic solution; coincidence degree theory; exponential stability; Krichhoff's matrix tree theorem; time-varying delays

\section{Introduction}

Differential and difference dynamic models have been intensively investigated because of their significance and applications in areas such as physics, mathematical biology and artificial neural networks [1-12]. In 1988, Bart Kosko first proposed the Bidirectional associative memory neural networks (BAMNNs) [13], a class of Recurrent neural networks which consist of two layers of neurons, namely U-layer and V-layer; neurons in the U-layer are fully interconnected with the neuron in the V-layer, moreover the connections between the neurons are no more when they are in the same layer. Due to its potential applications in optimization [14,15], associative memories [16,17], signal processing [18], pattern recognition [19], and so forth, BAM type NNs are attractive to many researchers [20-22]. Furthermore, time delays are unavoidable due to various reasons such as finite switching speed of amplifier circuit in electrical analog, sudden transmission of signals in NNs and so on [23-25]. Time delays are often encountered in different types of NNs like Hopfield neural networks [26], BAMNNs [27-31], inertial neural networks [32,33], cellular neural networks [34,35], complex neural networks, and so forth [36-38]. It may generate unwanted dynamical response such 
as stability, instability, oscillation, chaotic, periodic and so forth. Moreover, convergence analysis of BAMNNs have been a recent hot topic for research [21,39].

In practice, time delays are not mandatorily a constant; they may change over time and/or depending on system parameters [40-44]. It is pointed out that most of the studies on delayed neural networks (DNNs) have dealt with the stability issue of discrete time delay [21]. Moreover, NNs have a spatial nature because their parallel pathways have various sizes and lengths of axons, which lead the signal propagation to be no longer instantaneous but distributed during a certain period of time. This behavior can be modeled as distributed delay $[39,45]$. Furthermore, NNs are the mathematical analog of the human brain or biological NNs. Due to the potential application, NNs were applied in the field of speech recognition, optimization, characteristic recognition, control, time series prediction and so on. In the past few decades, NNs have attracted the considerable attention of researchers, among them most of the considered results and works are in the sense of continuous time and few of them are in discrete time [46]. Moreover, discrete time NNs are described in the application perspective in the fields of engineering and more specifically, numerical simulation.

In many real-world phenomena, periodic motion is common, such as in the biological system, the human brain oscillates periodically, the changing of climates in four seasons, waves and vibrations, and so on. Recently, there has been an increasing number of researchers working on this periodicity of NNs [22,47-50]. Until now, in the study of stability analysis of the NNs, the Lyapunov functional approach played a vital role [51,52]. Moreover, the construction of Lyapunov functional for a large scale system is not an easy job. To overcome this computational complexity, Li et al. [53] proposed the novel graph-theoretic approach. The main advantage of this work is to construct a global Lyapunov function for the large scale systems that are more related to the topological structure. Utilizing the achievements of the pioneering works, a few researchers have initiated their work and applied this approach to their research. For example, in Reference [54], the boundedness of the stochastic van der Pol oscillators was studied by using graph theory and the Lyapunov functional method; in Reference [55], the boundedness of the stochastic differential equations were studied by utilizing the novel approach; in Reference [56] the stability of neural networks was studied with the help of the graph-theoretic approach. However, the graph-theoretic approach is frequently used in the study of stability.

Motivated by the aforementioned works, we investigated the discrete time BAMNNs with mixed time-varying delays which are exponentially stable and have a unique periodic solution. The main contributions of this work are given below:

- According to the survey, there are few works on the exponential stability of periodic solution for discrete time delayed BAMNNs(DDBAMNNs).

- In this manuscript, together with the discrete and continuously distributed delay, the existence and periodic solution of discrete time BAMNNs is firstly proposed in the base of the graph theoretic approach, which generalizes and improves on the existing literature.

- To avoid the complication of finding the Lyapunov function, we construct a suitable Lyapunov function for a vertex system by using the results from graph theory.

- With the help of Lyapunov-Krasovkii functional and coincidence degree theory two types of sufficient criteria are derived that are different from the various techniques in previous works $[22,49,57,58]$.

The rest of this work is organized as follows: in the next section we give the model description of the proposed DDBAMNNs after which we provide the lemma, definition, and assumptions which are used throughout this work. In Section 3, the existence of periodic solutions for DDBAMNNs is achieved by employing the combination of degree theory and Kirchhoff's matrix tree theorem. In Section 4, by constructing the suitable Lyapunov function and the periodic solution conditions, the exponential stability of discrete-time NNs is derived. At the end of this work, to show the exactness of this work, we present a numerical simulation. 


\section{Preliminaries and Model Description}

In this work, the set of all positive integers, non-negative integers and n-dimensional Euclidean space are respectively indicated by $\mathbb{Z}^{+}, \mathbb{Z}_{0}^{+}$and $\mathbb{R}^{n}, \mathbb{R}^{m \times n}$ be denote the set of all $m \times n$ real matrices. The sets $S_{T}=\{0,1,2, \ldots, T-1\}$ and $\mathbb{S}=\{1,2, \ldots, n\}$. The difference operator of $f(x)$ be defined as $\Delta f(w)=f(w+1)-f(w)$. Denote $k, T \in \mathbb{Z}_{0}^{+},[a, b]_{\mathbb{Z}}=\{a, a+1, \ldots, b-1, b\},|$.$| denotes the$ Euclidean norm.

Graph theory [59]. A digraph $\mathbf{G}=(\mathbf{V}, \mathbf{A})$ with a set of all vertices or nodes $\mathbf{V}=\{1,2, \ldots, l\}$ as a neuron and directed edges $\mathbf{A} \subseteq \mathbf{V} \times \mathbf{V}$ the connection between them. A subgraph $\mathbf{H}=\left(\mathbf{V}_{H}, \mathbf{A}_{H}\right)$ of a digraph $\mathbf{G}$, if it has a same number of vertex set, that is, $\mathbf{V}=\mathbf{V}_{H}$ then it is known as a spanning subgraph of $\mathbf{G}$. If each arc $(k, h)$ is assigned by a positive weight $p_{k h}$ then the digraph $\mathbf{G}$ is said to be weighted digraph $\mathbf{W}(\mathbf{G})$. A set of all arcs of the digraph $\mathbf{G}$ with distinct vertices $\left\{P_{1}, P_{2}, \ldots, P_{k}\right\}$ is said to be a directed path $\mathbf{P}$ if $\left\{\left(P_{k}, P_{k+1}\right): k=1,2,3, \ldots, l-1\right\}$. The directed path $\mathbf{P}$ is said to be a directed cycle $\mathbf{Q}$ if $P_{k}=P_{1}$. A unicyclic graph $\mathbf{U}$ in digraph $\mathbf{G}$ is a subgraph with a disjoint union of rooted trees whose roots form a dicycle. We define the weighted matrix $Z=\left(z_{k h}\right)_{n \times n}$ of given weighted digraph $\mathbf{W}$ with 1 vertices whose entry $z_{k h}>0$ if the weight of $\operatorname{arc}(k, h)$ exists, otherwise 0 . $\mathbf{G}$ is said to be strongly connected whenever for any two of distinct vertices, there is a dipath to each other. The Laplacian matrix $\mathbf{L}_{p}$ of $(\mathbf{G}, \mathbf{A})$ is defined by $-l_{k h}$ for $k \neq h$ and if $k=h$ then it must be equal to $\sum_{i \neq k} l_{k i}$.

Coincidence degree theory [60]. Let $V$ and $W$ be respectively the normed vector spaces, the linear mapping $\mathcal{L}:$ dom $\mathcal{L} \rightarrow W$ satisfies $\operatorname{dim} \operatorname{ker} \mathcal{L}=\operatorname{codim} \operatorname{ker} \operatorname{Im} \mathcal{L}<\infty$ and $\operatorname{Im} \mathcal{L}$ is closed in $W$, is called the fredholm mapping of index zero, which implies that there exist projection $\mathcal{P}: V \rightarrow V$ and $\mathcal{Q}: W \rightarrow W$ which are continuous such that $\operatorname{Im} \mathcal{P}=\operatorname{ker} \mathcal{L}$ and $\operatorname{Im} \mathcal{L}=\operatorname{ker} Q=\operatorname{Im}(I-\mathcal{Q})$, which implies that $\mathcal{L} / \operatorname{dom} \mathcal{L} \cup \operatorname{ker} \mathcal{P}:(I-\mathcal{P}) V \rightarrow V$ is invertible, $K_{p}: \operatorname{Im} \mathcal{L} \rightarrow \operatorname{ker} P$ and we denote $\mathcal{L}_{p}^{-1}$ by $K_{p}$. If $\Theta$ is an open bounded subset of $\mathrm{V}$, the mapping $\mathcal{N}$ will be called $\mathcal{L}$-Compact on $\bar{\Theta}$ if $Q N \bar{\Theta}$ is bounded and $K_{p}(\mathcal{I}-\mathcal{Q}) \mathcal{N}: \bar{\Theta} \rightarrow V$ is compact, that is, $K_{\mathcal{P}, \mathcal{Q}}=K_{\mathcal{P}}(I-Q)$. Since, $\operatorname{Im} \mathcal{Q} \cong \operatorname{ker} \mathcal{L}$, there exists an isomorphism $\mathcal{J}: \operatorname{Im} \mathcal{Q} \rightarrow \operatorname{ker} \mathcal{L}$.

A mathematical description of BAM Neural Networks are considered as follows:

$$
\begin{aligned}
& u_{k}(i+1)=-a_{k} u_{k}(i)+\sum_{h=1}^{l} b_{k h} f_{h}\left(v_{h}(i)\right)+I_{k} \\
& v_{k}(i+1)=-p_{k} v_{k}(i)+\sum_{h=1}^{l} q_{k h} g_{h}\left(u_{h}(i)\right)+J_{k}, h=1,2,3, \ldots l
\end{aligned}
$$

for $l \geq 2$ to be the number of neurons in the first and second layers respectively; here, for $k \in Z_{0}^{+}, u_{k}(i)$ and $v_{k}(i) \in \mathbb{R}^{n}$ denotes the position of kth neuron at time $i \in\{1,2, \ldots, m\} \in \mathbb{Z}^{+}$in both layers and $\left(u_{k}(i), v_{k}(i)\right)=w_{k}(i) ; A=\operatorname{diag}\left\{a_{1}, a_{2}, \ldots, a_{n}\right\}>0$ and $P=\operatorname{diag}\left\{p_{1}, p_{2}, \ldots, p_{n}\right\}>0$ be the self-feedback matrices; in both layers $B=\left(b_{k h}\right)_{l \times l}$ and $Q=\left(q_{k h}\right)_{l \times l}$ in $\mathbb{R}^{n \times m}$ which stands for the connection weight matrices between kth and hth neuron; $f_{h}: \mathbb{R}^{n} \times \mathbb{Z}^{+} \rightarrow \mathbb{R}^{n}$ and $g_{h}: \mathbb{R}^{n} \times \mathbb{Z}^{+} \rightarrow \mathbb{R}^{n}$ be denotes the neuronal activation functions and the output functions are represented by $I_{k}$ and $J_{k}$.

As mentioned in the introduction, we introduce the discrete and continuously distributed delay in BAM Neural Networks:

$$
\begin{aligned}
u_{k}(i+1)= & -a_{k} u_{k}(i)+\sum_{h=1}^{l} b_{k h} f_{1}\left(v_{h}(i)\right)+\sum_{h=1}^{l} d_{k h} f_{2}\left(v_{h}\left(i-\tau_{k h}(i)\right)\right) \\
& +\sum_{h=1}^{l} e_{k h} f_{3}\left(\sum_{v=1}^{\infty} M_{k h}(v) v_{h}(i-v)\right)+I_{k}
\end{aligned}
$$




$$
\begin{aligned}
v_{k}(i+1)= & -p_{k} v_{k}(i)+\sum_{h=1}^{l} q_{k h} g_{1}\left(u_{h}(i)\right)+\sum_{h=1}^{l} r_{k h} g_{2}\left(u_{h}\left(i-\varsigma_{k h}(i)\right)\right) \\
& +\sum_{h=1}^{l} s_{k h} g_{3}\left(\sum_{\vartheta=1}^{\infty} O_{k h}(\vartheta) u_{h}(i-\vartheta)\right)+J_{k} ; \quad k=1,2,3, \ldots, l
\end{aligned}
$$

with Initial conditions,

$$
\begin{aligned}
& u_{k}(j)=\psi(j), \quad \forall j \in[-\tau, 0]_{\mathbb{Z}}, \quad \tau=\max _{1 \leq h \leq l}\left\{\tau_{k h}\right\} \\
& v_{k}(j)=\chi(j), \quad \forall j \in[-\varsigma, 0]_{\mathbb{Z},} \quad \varsigma=\max _{1 \leq h \leq l}\left\{\varsigma_{k h}\right\}
\end{aligned}
$$

where the set of real valued map $\psi:[-\tau, 0]_{\mathbb{Z}} \rightarrow \mathbb{R}^{n}$ and $\chi:[-\varsigma, 0]_{\mathbb{Z}} \rightarrow \mathbb{R}^{n}$. Here, $\tau(i), \varsigma(i)$ and $v, \vartheta$ represent respectively the discrete and distributed delay in both layers with $0 \leq \tau_{k h} \leq \tau, 0 \leq \varsigma_{k h} \leq \varsigma_{\text {; }}$ $D=\left(d_{k h}\right)_{l \times l}$ and $R=\left(r_{k h}\right)_{l \times l} \in \mathbb{R}^{m \times n}$ denotes the connection weight matrices of the discrete activation function $f_{2 h}\left(v_{h}\left(i-\tau_{k h}(i)\right)\right)$ and $g_{2 h}\left(v_{h}\left(i-\varsigma_{k h}(i)\right)\right) ; E=\left(e_{k h}\right)_{l \times l}$ and $S=\left(s_{k h}\right)_{l \times l}$ denotes the connection weight matrices of the infinitely distributed activation function $f_{3 h}\left(\sum_{v=1}^{\infty} M_{k h}(v) v_{h}(i-\right.$ $v)$ ) and $g_{3 h}\left(\sum_{\vartheta=1}^{\infty} O_{k h}(\vartheta) v_{h}(i-\vartheta)\right)$; where $M_{k h}$ and $O_{k h}$ stands for the kernel function.

\section{Assumption}

$\left(A_{1}\right)$ For any $h \in \mathbb{S}$ and a continuous and bounded function $f_{h}\left(v_{h}().\right)$ and $g_{h}\left(u_{h}().\right)$ there exist constants $L_{h}, N_{h}$ such that

$$
\begin{aligned}
\left|f_{h}\left(v_{h}(.)\right)\right| & \leq L_{h}\left|v_{h}(.)\right| \\
\left|g_{h}\left(u_{h}(.)\right)\right| & \leq N_{h}\left|u_{h}(.)\right|, \quad h=1,2,3 .
\end{aligned}
$$

$\left(A_{2}\right)$ The Kernel function $M_{k h}(v), O_{k h}(\vartheta) \in \mathbb{R}^{+}, \forall v, \vartheta \in \mathbb{Z}$ is bounded.

$\left(A_{3}\right) \sum_{v=1}^{\infty} M_{k h}(v)=1$ and $\sum_{\vartheta=1}^{\infty} N_{k h}(\vartheta)=1$

Lemma 1. See Reference [53] for $l \geq 2$ then the upcoming identity holds

$$
\sum_{k, h=1}^{l} c_{k} a_{k h} F_{k h}\left(w_{k}(i), w_{h}(i), i\right)=\sum_{\mathbf{U} \in \mathbf{U}} \mathbf{W}(\mathbf{U}) \sum_{(k, h) \in \mathbf{A}\left(\mathbf{Q}_{\mathbf{U}}\right)} F_{k h}\left(w_{k}(i), w_{h}(i), i\right) .
$$

Here, for any $k, h \in \mathbb{S}$, the cofactor of the $k$ th diagonal element of the Laplacian matrix is expressed as $c_{k}, F_{k h}\left(w_{k}(i), w_{h}(i), i\right)$ is an arbitrary function, $\mathbf{U}$ is the collection of all spanning unicyclic graphs of $(\mathbf{G}, \mathbf{A})$, $\mathbf{W}(\mathbf{U})$ is the weight of $\mathbf{U}$ and $\mathbf{Q}_{\mathbf{U}}$ denotes the directed cycle of $\mathbf{Q}$. In addition, $c_{k}>0$ whenever $(\mathbf{G}, \mathbf{A})$ is strongly connected.

Lemma 2 ([60]). The Fredholm mapping $\mathcal{L}$ of index zero and $\mathcal{L}$-Compact $\mathcal{N}$ on $\Theta$. Suppose

1. $\mathcal{L} w \neq \Lambda \mathcal{L} w$, for all $w \in \partial \Theta \cap \operatorname{ker} \mathcal{L}$ and $\Lambda \in(0,1)$.

2. $\mathcal{Q N} w \neq 0$, for all $w \in \partial \Theta \cap \operatorname{ker} \mathcal{L}$.

3. $\operatorname{deg}_{\mathcal{B}} \mathcal{J} \mathcal{Q N}, \Theta \cap \operatorname{ker} L, 0 \neq 0$. where deg denotes the Brouwer degree.

which implies that $\mathcal{L} w=\mathcal{N} w$ has at least one solution lying in $D o m \mathcal{L} \cap \bar{\Theta}$.

\section{The Existence of Periodic Solution for DBAMNNs}

In this section, for the given delayed BAM neural networks we derive the sufficient condition for the existence of a periodic solution by using the continuation theorem and Krichhoff's matrix tree theorem. 
Theorem 1. Let us consider the following assumptions are true:

$\left(P_{1}\right)$ There exists a Lyapunov function $v_{k}\left(w_{k}(i), i\right)$ such that

$$
v_{k}\left(w_{k}(i), i\right)=v_{k}\left(w_{k}(i+T)\right), \quad \lim _{\left|w_{k}\right| \rightarrow \infty} v_{k}\left(w_{k}, i\right)=\infty
$$

$\left(P_{2}\right)$ There exist the constants $\sigma_{k}, \gamma_{k}, \delta_{k}>0$ and the matrix $\left(\alpha_{k h}\right)_{l \times l}$, the arbitrary function $F_{k h}\left(w_{k}(i), w_{h}(i), i\right)$, for any $\lambda \in(0,1)$ such that

$$
\Delta v_{k}\left(w_{k}(i), i\right) \leq-\sigma_{k} v_{k}\left(w_{k}(i), i\right)+\gamma_{k} v_{k}\left(w_{k}\left(i-\omega_{k h}(i)\right), i\right)+\sum_{h=1}^{l} \alpha_{k h} F_{k h}\left(w_{k}(i), w_{h}(i), i\right)+\delta_{k}
$$

where $\omega_{k h}(i)=\max _{k, h}\left\{\tau_{k h}(i), \zeta_{k h}(i)\right\}$.

$\left(P_{3}\right)$ Along with each dicycle $\mathbf{C}$ of a weighted strongly connected directed graph $(\mathbf{G}, \mathbf{A})$ such that

$$
\sum_{(p, q) \in E\left(\mathbf{C}_{\mathbf{Q}}\right)} F_{p q}\left(w_{k}(i), w_{h}(i), i\right) \leq 0
$$

$\left(P_{4}\right)$ Suppose that

$$
\left(\begin{array}{c}
-a_{1} u_{1}(i)+\sum_{h=1}^{l} b_{1 h} f_{1}\left(v_{h}(i)\right)+\sum_{h=1}^{l} d_{1 h} f_{2}\left(v_{h}\left(i-\tau_{k h}(i)\right)\right)+\sum_{h=1}^{l} e_{1 h} f_{3}\left(\sum_{v=1}^{\infty} M_{1 h}(v) v_{h}(i-v)\right)+I_{1}(t) \\
\vdots \\
-a_{n} u_{n}(i)+\sum_{h=1}^{l} b_{n h} f_{1}\left(v_{h}(i)\right)+\sum_{h=1}^{l} d_{h h} f_{2}\left(v_{h}\left(i-\tau_{k h}(i)\right)\right)+\sum_{h=1}^{l} e_{n h} f_{3}\left(\sum_{v=1}^{\infty} M_{n h}(v) v_{h}(i-v)\right)+I_{n}(t) \\
-p_{1} v_{1}(i)+\sum_{h=1}^{l} q_{1 h} g_{1}\left(u_{h}(i)\right)+\sum_{h=1}^{l} r_{1 h} g_{2}\left(u_{h}\left(i-\varsigma_{k h}(i)\right)\right)+\sum_{h=1}^{l} e_{1 h} g_{3}\left(\sum_{\vartheta=1}^{\infty} M_{1 h}(\vartheta) u_{h}(i-\vartheta)\right)+J_{1}(t) \\
\vdots \\
-p_{n} v_{n}(i)+\sum_{h=1}^{l} q_{n h} g_{1}\left(u_{h}(i)\right)+\sum_{h=1}^{l} r_{n h} g_{2}\left(u_{h}\left(i-\varsigma_{k h}(i)\right)\right)+\sum_{h=1}^{l} e_{n h} g_{3}\left(\sum_{\vartheta=1}^{\infty} M_{n h}(\vartheta) u_{h}(i-\vartheta)\right)+J_{n}(t)
\end{array}\right)=\mathcal{S}(w) w
$$

where $(\mathrm{S}(w))_{2 n \times 2 n}$ is a non-singular matrix. Then (2) has at least one T-Periodic solution.

Proof. For notation convenience denote

$$
w(i)=\left(w_{1}(i), w_{2}(i), \ldots, w_{n}(i)\right)^{T}=\left(u_{1}(i), u_{2}(i), \ldots, u_{n}(i), v_{1}(i), v_{2}(i), \ldots, v_{n}(i)\right)^{T} \in \mathbb{R}^{2 n}
$$

Let us consider

$$
V=W=\left\{w=\{w(i)\}: w(i) \in \mathbb{R}^{2 n}, n \in \mathbb{N}\right\}=p^{m}
$$

and $p^{T} \subset p^{m}$ which denotes the subspace of all T-periodic sequence equipped with norm

$$
\|w\|=\sum_{k=1}^{l}\left(\max _{i \in S_{T}}\left|u_{k}(i)\right|+\max _{i \in S_{T}}\left|v_{k}(i)\right|\right)
$$

for any $w(i) \in p^{T}$. Clearly, $p^{T}$ is a finite dimensional Banach space. Define a map $\mathcal{L}:$ Dom $\mathcal{L} \subset V \rightarrow V$ and $\mathcal{N}: V \rightarrow V$ by $\mathcal{L} w(i)=\Delta \mathcal{N} w(i)$ and 


$$
\begin{aligned}
\mathcal{N} w(i)=\mathcal{N}\left(\begin{array}{c}
w_{1}(i) \\
w_{2}(i) \\
\vdots \\
w_{h}(i)
\end{array}\right) \\
=\left[\begin{array}{c}
-a_{1} u_{1}(i)+\sum_{h=1}^{l} b_{1 h} f_{1}\left(v_{h}(i)\right)+\sum_{h=1}^{l} d_{1 h} f_{2}\left(v_{h}\left(i-\tau_{k h}(i)\right)\right)+\sum_{h=1}^{l} e_{1 h} f_{3}\left(\sum_{v=1}^{\infty} M_{1 h}(v) v_{h}(i-v)\right)+I_{1}(t) \\
\vdots \\
-a_{n} u_{n}(i)+\sum_{h=1}^{l} b_{n h} f_{1}\left(v_{h}(i)\right)+\sum_{h=1}^{l} d_{n h} f_{2}\left(v_{h}\left(i-\tau_{k h}(i)\right)\right)+\sum_{h=1}^{l} e_{h h} f_{3}\left(\sum_{v=1}^{\infty} M_{n h}(v) v_{h}(i-v)\right)+I_{n}(t) \\
-p_{1} v_{1}(i)+\sum_{h=1}^{l} q_{1 h} g_{1}\left(u_{h}(i)\right)+\sum_{h=1}^{l} r_{1 h} g_{2}\left(u_{h}\left(i-\varsigma_{k h}(i)\right)\right)+\sum_{h=1}^{l} e_{1 h} g_{3}\left(\sum_{\vartheta=1}^{\infty} M_{1 h}(\vartheta) u_{h}(i-\vartheta)\right)+J_{1}(t) \\
\vdots \\
-p_{n} v_{n}(i)+\sum_{h=1}^{l} q_{n h} g_{1}\left(u_{h}(i)\right)+\sum_{h=1}^{l} r_{n h} g_{2}\left(u_{h}\left(i-\varsigma_{k h}(i)\right)\right)+\sum_{h=1}^{l} e_{h h} g_{3}\left(\sum_{\vartheta=1}^{\infty} M_{n h}(\vartheta) u_{h}(i-\vartheta)\right)+J_{n}(t)
\end{array}\right]
\end{aligned}
$$

Then,

$$
\begin{aligned}
\operatorname{Im} \mathcal{L} & =\left\{w=\{w(i)\} \in p^{T}: \sum_{i=0}^{T-1} w(i)=0\right\} \\
\operatorname{Ker} \mathcal{L} & =\left\{w=\{w(i)\} \in p^{T}: w(i)=\text { constant } \in \mathbb{R}^{2 n}, i \in \mathbb{Z}\right\}
\end{aligned}
$$

which is closed in $W$ and $\operatorname{dim} \operatorname{Ker} \mathcal{L}=2 n=\operatorname{dim} \operatorname{Im} \mathcal{L}<+\infty$. It is easy to verify that $\operatorname{Im} \mathcal{L}$ and $\operatorname{Ker} \mathcal{L}$ are a closed linear subspace of $p^{T}$ and

$$
p^{T}=\operatorname{Ker} \mathcal{L} \oplus \operatorname{Im} \mathcal{L}
$$

Since $\operatorname{Im} \mathcal{L}$ is closed in $W$ and it has a finite dimensional, hence $\mathcal{L}$ is a Fredholm mapping of index zero. Let us define a projector $\mathcal{P}$ and $\mathcal{Q}$ as follows, $\mathcal{P}: V \cap \operatorname{Dom} \mathcal{L} \rightarrow \operatorname{ker} \mathcal{L}$ and $\mathcal{Q}: V \rightarrow V / \operatorname{Im} \mathcal{L}$.

$$
\begin{gathered}
\mathcal{P} w=\frac{1}{T} \sum_{i=0}^{T-1} w(i), \quad \mathcal{Q} w=\frac{1}{T} \sum_{i=0}^{T-1} w(i), \forall w \in V \\
\mathcal{P} w=\mathcal{Q} w=\frac{1}{T}\left(\begin{array}{c}
\sum_{i=0}^{T-1} w_{1}(i) \\
\sum_{i=0}^{T-1} w_{2}(i) \\
\vdots \\
\sum_{i=0}^{T-1} w_{n}(i)
\end{array}\right)
\end{gathered}
$$

Hence,

$$
\operatorname{Im} \mathcal{P}=\operatorname{Ker} \mathcal{L}, \operatorname{Im} \mathcal{L}=\operatorname{Ker} \mathcal{Q}=\operatorname{Im}(I-\mathcal{Q}) .
$$

Furthermore, the generalized inverse of $L, K_{p}: \operatorname{Im} \mathcal{L} \rightarrow \operatorname{Ker} \mathcal{P} \cap D o m \mathcal{L}$. is defined as

$$
K_{p}(w)=\sum_{i=0}^{T-1} w(i)-\frac{1}{T} \sum_{i=0}^{T-1}(T-i) w(i)
$$


Clearly,

$$
\mathcal{Q N} w=\left[\begin{array}{c}
\frac{1}{T} \sum_{i=0}^{T-1}\left[-a_{1} u_{1}(i)+\sum_{h=1}^{l} b_{1 h} f_{1}\left(v_{h}(i)\right)+\sum_{h=1}^{l} d_{1 h} f_{2}\left(v_{h}\left(i-\tau_{k h}(i)\right)\right)+\sum_{h=1}^{l} e_{1 h} f_{3}\left(\sum_{v=1}^{\infty} M_{1 h}(v) v_{h}(i-v)\right)+I_{1}(t)\right] \\
\vdots \\
\frac{1}{T} \sum_{i=0}^{T-1}\left[-a_{n} u_{n}(i)+\sum_{h=1}^{l} b_{h h} f_{1}\left(v_{h}(i)\right)+\sum_{h=1}^{l} d_{n h} f_{2}\left(v_{h}\left(i-\tau_{k h}(i)\right)\right)+\sum_{h=1}^{l} e_{h h} f_{3}\left(\sum_{v=1}^{\infty} M_{n h}(v) v_{h}(i-v)\right)+I_{n}(t)\right] \\
\frac{1}{T} \sum_{i=0}^{T-1}\left[-p_{1} v_{1}(i)+\sum_{h=1}^{l} q_{1 h} g_{1}\left(u_{h}(i)\right)+\sum_{h=1}^{l} r_{1 h} g_{2}\left(u_{h}\left(i-\varsigma_{k h}(i)\right)\right)+\sum_{h=1}^{l} e_{1 h} g_{3}\left(\sum_{\vartheta=1}^{\infty} M_{1 h}(\vartheta) u_{h}(i-\vartheta)\right)+J_{1}(t)\right] \\
\vdots \\
\frac{1}{T} \sum_{i=0}^{T-1}\left[-p_{n} v_{h}(i)+\sum_{h=1}^{l} q_{n h} g_{1}\left(u_{h}(i)\right)+\sum_{h=1}^{l} r_{n h} g_{2}\left(u_{h}\left(i-\varsigma_{k h}(i)\right)\right)+\sum_{h=1}^{l} e_{n h} g_{3}\left(\sum_{\vartheta=1}^{\infty} M_{n h}(\vartheta) u_{h}(i-\vartheta)\right)+J_{n}(t)\right]
\end{array}\right]
$$

and $K_{p}(I-\mathcal{Q}) \mathcal{N} w$ are continuous. Since $V$ and $W$ are finite dimensional Banach space and $K_{p}(I-\mathcal{Q}) \mathcal{N}$ is continuous. By using Ascoli-Arzela's theorem we can show that $\mathcal{Q N} \overline{(\Theta)}$ and $K_{p}(I-\mathcal{Q}) \mathcal{N} \bar{\Theta}$ are relatively compact for any open bounded set $\Theta \in V$. Hence $\mathcal{N}$ is compact on $\bar{\Theta}$. In the view of (1) the operation equation

$$
\mathcal{L} w=\Lambda \mathcal{N}
$$

for some $\Lambda \in(0,1)$.

$$
\Delta w(i)=\Lambda\left[\begin{array}{c}
-\left(1+a_{1}\right) u_{1}(i)+\sum_{h=1}^{l} b_{1 h} f_{1}\left(v_{h}(i)\right)+\sum_{h=1}^{l} d_{1 h} f_{2}\left(v_{h}\left(i-\tau_{k h}(i)\right)\right)+\sum_{h=1}^{l} e_{1 h} f_{3}\left(\sum_{v=1}^{\infty} M_{1 h}(v) v_{h}(i-v)\right)+I_{1}(t) \\
\vdots \\
-\left(1+a_{h}\right) u_{n}(i)+\sum_{h=1}^{l} b_{n h} f_{1}\left(v_{h}(i)\right)+\sum_{h=1}^{l} d_{n h} f_{2}\left(v_{h}\left(i-\tau_{k h}(i)\right)\right)+\sum_{h=1}^{l} e_{n h} f_{3}\left(\sum_{v=1}^{\infty} M_{n h}(v) v_{h}(i-v)\right)+I_{n}(t) \\
-\left(1+p_{1}\right) v_{1}(i)+\sum_{h=1}^{l} q_{1 h} g_{1}\left(u_{h}(i)\right)+\sum_{h=1}^{l} r_{1 h} g_{2}\left(u_{h}\left(i-\zeta_{k h}(i)\right)\right)+\sum_{h=1}^{l} e_{1 h} g_{3}\left(\sum_{\vartheta=1}^{\infty} M_{1 h}(\vartheta) u_{h}(i-\vartheta)\right)+J_{1}(t) \\
\vdots \\
-\left(1+p_{h}\right) v_{n}(i)+\sum_{h=1}^{l} q_{n h} g_{1}\left(u_{h}(i)\right)+\sum_{h=1}^{l} r_{h h} g_{2}\left(u_{h}\left(i-\zeta_{k h}(i)\right)\right)+\sum_{h=1}^{l} e_{n h} g_{3}\left(\sum_{\vartheta=1}^{\infty} M_{n h}(\vartheta) u_{h}(i-\vartheta)\right)+J_{n}(t)
\end{array}\right]
$$

Let us consider the global Lyapunov function

$$
V(w(i))=\sum_{k=1}^{l} c_{k} v_{k}\left(w_{k}(i)\right)
$$

where $c_{k}$ indicate the cofactor of $k$ th diagonal element of $\mathbf{L}_{p}$ and

$$
\begin{aligned}
\Delta V(w)= & \sum_{k=1}^{l} c_{k} \Delta v_{k}\left(w_{k}(i)\right) \\
\leq & \Lambda \sum_{k=1}^{l} c_{k}\left[-\sigma_{k}\left|w_{k}(i)\right|^{2}+\alpha_{k}\left|w_{k}\left(i-\omega_{k h}(i)\right)\right|^{2}+\sum_{h=1}^{l} F_{k h}\left(w_{k}(i), w_{h}(i)\right)+\delta_{k}\right] \\
\leq & \Lambda \sum_{k=1}^{l} c_{k}\left[-\frac{\sigma_{k}}{\eta_{k}} V_{k}\left(w_{k}(i)\right)+\frac{\alpha_{k}}{\gamma_{k}} V_{k}\left(w_{k}\left(i-\omega_{k h}(i)\right)\right)+\sum_{h=1}^{l} F_{k h}\left(w_{k}(i), w_{h}(i)\right)+\delta_{k}\right] \\
\leq & -\Lambda \sum_{k=1}^{l} c_{k} \frac{\sigma_{k}}{\eta_{k}} V_{k}\left(w_{k}(i)\right)+\Lambda \sum_{k, h=1}^{l} c_{k} \frac{\alpha_{k}}{\gamma_{k}} V_{k}\left(w_{k}\left(i-\omega_{k h}(i)\right)\right)+\Lambda \sum_{k=1}^{l} c_{k} F_{k h}\left(w_{k}(i), w_{h}(i)\right) \\
& +\Lambda \sum_{k=1}^{l} c_{k} \delta_{k} .
\end{aligned}
$$


where, $w(i)$ is T-periodic solution which implies $V(w(i))$ is also a T-periodic function.

From assumption

$$
\begin{aligned}
0 \leq & -\Lambda \sum_{k=1}^{l} c_{k} \frac{\sigma_{k}}{\eta_{k}} \sum_{i=0}^{T-1} V_{k}\left(w_{k}(i)\right)+\Lambda \sum_{k=1}^{l} c_{k} \frac{\alpha_{k}}{\gamma_{k}} \sum_{i=0}^{T-1} V_{k}\left(w_{k}\left(i-\omega_{k h}(i)\right)\right)+\Lambda \sum_{k, h=1}^{l} c_{k} \\
& \times \sum_{i=0}^{T-1} F_{k h}\left(w_{k}(i), w_{h}(i)\right)+\Lambda T \sum_{k=1}^{l} c_{k} \delta_{k}
\end{aligned}
$$

$$
\begin{aligned}
& \leq-\Lambda \sum_{k=1}^{l} c_{k} \frac{\sigma_{k}}{\eta_{k}} \sum_{i=0}^{T-1} V_{k}\left(w_{k}(i)\right)+\Lambda \sum_{k=1}^{l} c_{k} \frac{\alpha_{k}}{\gamma_{k}} \sum_{i=0}^{T-1} V_{k}\left(w_{k}\left(i-\omega_{k h}(i)\right)\right)+\Lambda T \sum_{k=1}^{l} c_{k} \delta_{k} \\
& \leq-\Lambda \sigma \sum_{i=0}^{T-1} V(w(i))+\Lambda \alpha \sum_{i=0}^{T-1} V\left(w\left(i-\omega_{k h}(i)\right)\right)+\Lambda T \sum_{k=1}^{l} c_{k} \delta_{k} \\
& <0 .
\end{aligned}
$$

Here,

$$
\begin{gathered}
c=\min _{k}\left\{c_{k}\right\} ; \sigma=\min _{k}\left\{\frac{\sigma_{k}}{\eta_{k}}\right\} ; \alpha=\min _{k}\left\{\frac{\alpha_{k}}{\gamma_{k}}\right\} ; \\
\lim _{w \rightarrow \infty} \Delta V(w)<0
\end{gathered}
$$

This contradicts a T-periodic function $V(w(i))$. Therefore, there exists $\mathcal{B}>0$ which is separate from the selection of $\Lambda$, so that the solution of $\mathcal{L} w=\Lambda w$, which satisfies $\|w\|<\mathcal{B}$. Denotes

$$
\Theta=\{w \in \mathcal{B}:\|w\|<\mathcal{B}\} .
$$

Then, we know that $\mathcal{L} w \neq \Lambda \mathcal{N} w, \Lambda \in(0,1)$ for $w \in \operatorname{Ker} \mathcal{L} \cap \partial \Theta$ So, we obtain

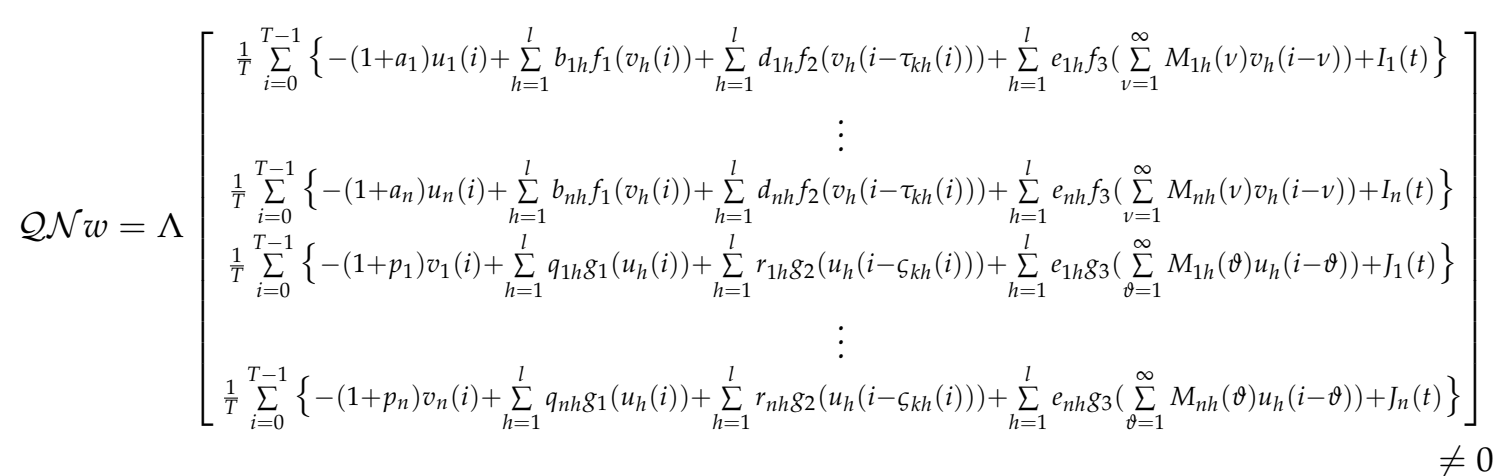

Let us define a identity mapping $\mathcal{I}: \operatorname{Im} \mathcal{Q} \rightarrow \operatorname{Ker} \mathcal{L}$. Then for any $(w, \mu) \in \Theta \in[0,1]$.

$$
\mathcal{I} \mathcal{Q N} w=\left[\begin{array}{c}
\frac{1}{T} \sum_{i=0}^{T-1}\left\{-\left(1+a_{1}\right) u_{1}(i)+\sum_{h=1}^{l} b_{1 h} f_{1}\left(v_{h}(i)\right)+\sum_{h=1}^{l} d_{1 h} f_{2}\left(v_{h}\left(i-\tau_{k h}(i)\right)\right)+\sum_{h=1}^{l} e_{1 h} f_{3}\left(\sum_{v=1}^{\infty} M_{1 h}(v) v_{h}(i-v)\right)+I_{1}(t)\right\} \\
\vdots \\
\frac{1}{T} \sum_{i=0}^{T-1}\left\{-\left(1+a_{n}\right) u_{n}(i)+\sum_{h=1}^{l} b_{n h} f_{1}\left(v_{h}(i)\right)+\sum_{h=1}^{l} d_{h h} f_{2}\left(v_{h}\left(i-\tau_{k h}(i)\right)\right)+\sum_{h=1}^{l} e_{n h} f_{3}\left(\sum_{v=1}^{\infty} M_{n h}(v) v_{h}(i-v)\right)+I_{n}(t)\right\} \\
\frac{1}{T} \sum_{i=0}^{T-1}\left\{-\left(1+p_{1}\right) v_{1}(i)+\sum_{h=1}^{l} q_{1 h} g_{1}\left(u_{h}(i)\right)+\sum_{h=1}^{l} r_{1 h} g_{2}\left(u_{h}\left(i-\zeta_{k h}(i)\right)\right)+\sum_{h=1}^{l} e_{1 h} g_{3}\left(\sum_{\vartheta=1}^{\infty} M_{1 h}(\vartheta) u_{h}(i-\vartheta)\right)+J_{1}(t)\right\} \\
\vdots \\
\frac{1}{T} \sum_{i=0}^{T-1}\left\{-\left(1+p_{n}\right) v_{n}(i)+\sum_{h=1}^{l} q_{n h} g_{1}\left(u_{h}(i)\right)+\sum_{h=1}^{l} r_{n h} g_{2}\left(u_{h}\left(i-\varsigma_{k h}(i)\right)\right)+\sum_{h=1}^{l} e_{n h} g_{3}\left(\sum_{\vartheta=1}^{\infty} M_{n h}(\vartheta) u_{h}(i-\vartheta)\right)+J_{n}(t)\right\}
\end{array}\right]
$$


Define,

$$
\begin{aligned}
F(w, \mu)= & \mu w-(1-\mu) \mathcal{I} \mathcal{Q N} w, \quad(w, \mu) \in \Theta \times[0,1] . \\
=\mu & \left(\begin{array}{c}
w_{1}(i) \\
w_{2}(i) \\
\vdots \\
w_{n}(i)
\end{array}\right)-\frac{1-\mu}{T} \\
& {\left[\begin{array}{c}
\frac{1}{T} \sum_{i=0}^{T-1}\left\{-\left(1+a_{1}\right) u_{1}(i)+\sum_{h=1}^{l} b_{1 h} f_{1}\left(v_{h}(i)\right)+\sum_{h=1}^{l} d_{1 h} f_{2}\left(v_{h}\left(i-\tau_{k h}(i)\right)\right)+\sum_{h=1}^{l} e_{1 h} f_{3}\left(\sum_{v=1}^{\infty} M_{1 h}(v) v_{h}(i-v)\right)+I_{1}(t)\right\} \\
\vdots \\
\frac{1}{T} \sum_{i=0}^{T-1}\left\{-\left(1+a_{h}\right) u_{n}(i)+\sum_{h=1}^{l} b_{n h} f_{1}\left(v_{h}(i)\right)+\sum_{h=1}^{l} d_{n h} f_{2}\left(v_{h}\left(i-\tau_{k h}(i)\right)\right)+\sum_{h=1}^{l} e_{n h} f_{3}\left(\sum_{v=1}^{\infty} M_{n h}(v) v_{h}(i-v)\right)+I_{n}(t)\right\} \\
\frac{1}{T} \sum_{i=0}^{T-1}\left\{-\left(1+p_{1}\right) v_{1}(i)+\sum_{h=1}^{l} q_{1 h} g_{1}\left(u_{h}(i)\right)+\sum_{h=1}^{l} r_{1 h} g_{2}\left(u_{h}\left(i-\varsigma_{k h}(i)\right)\right)+\sum_{h=1}^{l} e_{1 h} g_{3}\left(\sum_{\vartheta=1}^{\infty} M_{1 h}(\vartheta) u_{h}(i-\vartheta)\right)+J_{1}(t)\right\} \\
\vdots \\
\frac{1}{T} \sum_{i=0}^{T-1}\left\{-\left(1+p_{n}\right) v_{n}(i)+\sum_{h=1}^{l} q_{n h} g_{1}\left(u_{h}(i)\right)+\sum_{h=1}^{l} r_{n h} g_{2}\left(u_{h}\left(i-\varsigma_{k h}(i)\right)\right)+\sum_{h=1}^{l} e_{n h} g_{3}\left(\sum_{\vartheta=1}^{\infty} M_{n h}(\vartheta) u_{h}(i-\vartheta)\right)+J_{n}(t)\right\}
\end{array}\right] }
\end{aligned}
$$

For $\mu \in(0,1)$.

$$
\operatorname{deg}_{\mathcal{B}}\{\mathcal{J} \mathcal{Q N}, \Theta \cap \operatorname{Ker} \mathcal{L}, 0\}=\operatorname{Sgn}(\operatorname{det}(G(0))) \neq 0
$$

Hence, all the conditions in the continuation theorem are satisfied. Then (2) has T-periodic solution.

Remark 1. Suppose that the digraph $(\mathbf{G}, \mathbf{A})$ is balanced, then the following equation possess

$$
\begin{aligned}
\sum_{k, h=1}^{l} a_{k h} F_{k h}\left(w_{k}(i), w_{h}(i)\right)= & \frac{1}{2} \sum_{\mathbf{U} \in \mathbb{U}} \mathbf{W}(\mathbf{U}) \sum_{(k, h) \in \mathbf{A}\left(\mathbf{Q}_{\mathbf{U}}\right)}\left[F_{k h}\left(w_{k}(i), w_{h}(i)\right)\right. \\
& \left.+F_{h k}\left(w_{h}(i), w_{k}(i)\right)\right] .
\end{aligned}
$$

Extending the condition, (5) into

$$
\sum_{(k, h) \in \mathbf{Q}_{\mathbf{U}}}\left[F_{k h}\left(w_{k}(i), w_{h}(i)\right)+F_{h k}\left(w_{h}(i), w_{k}(i)\right)\right] \leq 0 .
$$

Corollary 1. Let $(\mathbf{G}, \mathbf{A})$ be a balanced digraph. If we put (10) instead of (4) in the place of $a_{k h} F_{k h}\left(w_{k}(i), w_{h}(i)\right)$ then the system (2) has T-periodic solution.

Remark 2. For each $k, h$, there exists functions $R_{k}\left(w_{k}\right)$ and $R_{h}\left(w_{h}\right)$ such that

$$
F_{k h}\left(w_{k}(i), w_{h}(i)\right) \leq R_{k}\left(w_{k}(i)\right)-R_{h}\left(w_{h}(i)\right) .
$$

At that point

$$
\sum_{(k, h) \in \mathbf{Q}_{\mathbf{U}}}\left[R_{k}\left(w_{k}(i)\right)-R_{h}\left(w_{h}(i)\right)\right] \leq 0 .
$$

Corollary 2. The determination of existence theorem holds whenever we replace (4) by (11).

Remark 3. For DDBAMNNs (2), we construct a global Lyapunov function $V=\sum_{k=1}^{l} c_{k} v_{i}$, and that is closely connected to the topological structure. However, the construction of Lyapunov function for delayed system is a difficult one. Hence, we design $v_{i}=v_{k}^{(1)}(i)+v_{k}^{(2)}(i)+v_{k}^{(3)}(i)$, which is useful for solving this type problem. 


\section{Uniqueness and Exponential Stability of Periodic Solution}

Definition 1. The system (2) with T-periodic solution $u^{*}(i)$ and $v^{*}(i)$ is said to be exponentially stable if there is a constant $\kappa>0$ and $\rho>1$ such that

$$
\left|u(i)-u^{*}(i)\right|^{2}+\left|v(i)-v^{*}(i)\right|^{2} \leq \rho^{i} \kappa\left(\sup _{s \in \mathbb{Z}[-\tau, 0]}\left\|u(i)-u^{*}(i)\right\|^{2}+\sup _{s \in \mathbb{Z}[-\zeta, 0]}\left\|v(i)-v^{*}(i)\right\|^{2}\right)
$$

Moreover, exponentially stable implies the uniqueness of T-periodic solution $u^{*}(i)$ and $v^{*}(i)$.

Theorem 2. Let us consider the condition $\left(A_{1}\right)-\left(A_{3}\right)$, if

$$
\begin{aligned}
& \max \left\{\rho\left|a_{k}\right|^{2}+\rho \sum_{h=1}^{l} e_{k h}^{2} L_{3}^{2} \sum_{v=1}^{\infty} M_{k h}(v)+\rho \sum_{k=1}^{l}\left|d_{h k}\right|^{2} L_{2}^{2}+\rho \sum_{k=1}^{l}\left|q_{h k}\right|^{2} N_{1}^{2}, \rho\left|p_{k}\right|^{2}+\rho \sum_{h=1}^{l} s_{k h}^{2} N_{3}^{2}\right. \\
& \left.\times \sum_{\vartheta=1}^{\infty} O_{k h}(\vartheta)+\rho \sum_{k=1}^{l}\left|r_{h k}\right|^{2} N_{2}^{2}+\rho \sum_{k=1}^{l}\left|b_{h k}\right|^{2} L_{1}^{2}\right\}>1
\end{aligned}
$$

then the system (2) is exponential stability and it is unique.

Proof. On the basis of Theorem 1, the system (2) has at least one T-periodic solution. Let

$$
w^{*}(i)=\left(u_{1}^{*}(i), u_{2}^{*}(i), \ldots, u_{n}^{*}(i), v_{1}^{*}(i), v_{2}^{*}(i), \ldots, v_{n}^{*}(i)\right)^{T}
$$

be denotes the T-Periodic solution of (2). Then we have,

$$
\begin{aligned}
u_{k}^{*}(i+1)= & -a_{k} u_{k}^{*}(i)+\sum_{h=1}^{l} b_{k h} f_{1}\left(v_{h}^{*}(i)\right)+\sum_{h=1}^{l} d_{k h} f_{2}\left(v_{h}^{*}\left(i-\tau_{k h}(i)\right)\right) \\
& +\sum_{h=1}^{l} e_{k h} f_{3}\left(\sum_{v=1}^{\infty} M_{k h}(v) v_{h}^{*}(i-v)\right)+I_{k} \\
v_{k}^{*}(i+1)= & -p_{k} v_{k}^{*}(i)+\sum_{h=1}^{l} q_{k h} g_{1}\left(u_{h}^{*}(i)\right)+\sum_{h=1}^{l} r_{k h} g_{2}\left(u_{h}^{*}\left(i-\varsigma_{k h}(i)\right)\right) \\
& +\sum_{h=1}^{l} s_{k h} g_{3}\left(\sum_{\vartheta=1}^{\infty} O_{k h}(\vartheta) u_{h}^{*}(i-\vartheta)\right)+J_{k}
\end{aligned}
$$

for $i \in\{0,1, \ldots, T\}, k \in \mathbb{L}$.

Let

$$
x_{k}(i)=u_{k}(i)-u_{k}^{*}(i) \quad y_{k}(i)=v_{k}(i)-v_{k}^{*}(i)
$$


Then, we have

$$
\begin{aligned}
x_{k}^{*}(i+1)= & -a_{k} x_{k}^{*}(i)+\sum_{h=1}^{l} b_{k h}\left(f_{1}\left(v_{h}(i)\right)-f_{1}\left(v_{h}^{*}(i)\right)\right)+\sum_{h=1}^{l} d_{k h}\left(f_{2}\left(v_{h}\left(i-\tau_{k h}(i)\right)\right)\right. \\
& \left.-f_{2}\left(v_{h}^{*}\left(i-\tau_{k h}(i)\right)\right)\right)+\sum_{h=1}^{l} e_{k h}\left(f_{3}\left(\sum_{v=1}^{\infty} M_{k h}(v) v_{h}(i-v)\right)-f_{3}\left(\sum_{v=1}^{\infty} M_{k h}(v)\right.\right. \\
& \left.\left.\times v_{h}^{*}(i-v)\right)\right)+I_{k} \\
y_{k}^{*}(i+1)= & -p_{k} y_{k}^{*}(i)+\sum_{h=1}^{l} q_{k h}\left(g_{1}\left(u_{h}(i)\right)-g_{1}\left(u_{h}^{*}(i)\right)\right)+\sum_{h=1}^{l}\left(r_{k h} g_{2}\left(u_{h}\left(i-\varsigma_{k h}(i)\right)\right)\right. \\
& -r_{k h} g_{2}\left(u_{h}^{*}\left(i-\varsigma_{k h}(i)\right)\right)+\sum_{h=1}^{l} s_{k h}\left(g_{3}\left(\sum_{\vartheta=1}^{\infty} O_{k h}(\vartheta) u_{h}(i-\vartheta)\right)-g_{3}\left(\sum_{\vartheta=1}^{\infty} O_{k h}(\vartheta)\right.\right. \\
& \left.\left.\times u_{h}^{*}(i-\vartheta)\right)\right)+J_{k}
\end{aligned}
$$

Denote the Lyapunov function as $v_{k}(i)=v_{k}^{(1)}(i)+v_{k}^{(2)}(i)+v_{k}^{(3)}(i)$ and the cofactor of $k$ th diagonal element of $L_{p}$ is $c_{k}$. Let us define a global Lyapunov function

$$
V(i)=\sum_{k=1}^{l} c_{k} v_{k}(i)
$$

Here,

$$
\begin{aligned}
& v_{k}^{(1)}(i)=\frac{1}{2} \rho^{i}\left|x_{k}(i)\right|^{2}+\frac{1}{2} \rho^{i}\left|y_{k}(i)\right|^{2} \\
& v_{k}^{(2)}(i)=\frac{1}{2} \rho^{i} \sum_{h=1}^{l} d_{k h}^{2} L_{2}^{2} \sum_{\xi=i-\tau_{k h}(i)}^{i-1}\left|x_{h}(\xi)\right|^{2}+\frac{1}{2} \rho^{i} \sum_{h=1}^{l} r_{k h}^{2} N_{2}^{2} \sum_{\xi=i-\zeta_{k h}(i)}^{i-1}\left|y_{h}(\xi)\right|^{2} \\
& v_{k}^{(3)}(i)=\frac{1}{2} \rho^{i} \sum_{h=1}^{l} e_{k h}^{2} L_{3}^{2} \sum_{v=1}^{\infty} M_{k h}(v) \sum_{r=i-v}^{i-1}\left|x_{h}(r)\right|^{2}+\frac{1}{2} \rho^{i} \sum_{h=1}^{l} s_{k h}^{2} N_{3}^{2} \sum_{\vartheta=1}^{\infty} O_{k h}(\vartheta) \sum_{r=i-\vartheta}^{i-1}\left|y_{h}(r)\right|^{2}
\end{aligned}
$$


From Assumption I, we get

$$
\begin{aligned}
& \Delta v_{k}^{(1)}(i)=v_{k}^{(1)}(i+1)-v_{k}^{(1)}(i) \\
& =\frac{1}{2} \rho^{i}\left\{\rho x_{k}^{2}(i+1)-x_{k}^{2}(i)\right\}+\frac{1}{2} \rho^{i}\left\{\rho y_{k}^{2}(i+1)-y_{k}^{2}(i)\right\} \\
& =\frac{1}{2} \rho^{i}\left\{\rho \left(\mid-a_{k} x_{k}(i)+\sum_{h=1}^{l} b_{k h} f_{1}\left(v_{h}(i)\right)-\sum_{h=1}^{l} b_{k h} f_{1}\left(v_{h}^{*}(i)\right)+\sum_{h=1}^{l} d_{k h} f_{2}\left(v_{h}\left(i-\tau_{k h}(i)\right)\right)-\sum_{h=1}^{l} d_{k h}\right.\right. \\
& \left.\left.\times f_{2}\left(v_{h}^{*}\left(i-\tau_{k h}(i)\right)\right)+\sum_{h=1}^{l} e_{k h} f_{3} \sum_{v=1}^{\infty} M_{k h}(v) v_{h}(i-v)-\sum_{h=1}^{l} e_{k h} f_{3}\left(\sum_{v=1}^{\infty} M_{k h}(v) v_{h}^{*}(i-v)\right)+\left.I_{k}\right|^{2}\right)\right\} \\
& +\frac{1}{2} \rho^{i}\left\{\rho \left(\mid-p_{k} y_{k}^{*}(i)+\sum_{h=1}^{l} q_{k h} g_{1}\left(u_{h}(i)\right)-\sum_{h=1}^{l} q_{k h} g_{1}\left(u_{h}^{*}(i)\right)+\sum_{h=1}^{l} r_{k h} g_{2}\left(u_{h}\left(i-\varsigma_{k h}(i)\right)\right)-\sum_{h=1}^{l} r_{k h}\right.\right. \\
& \left.\times g_{2}\left(u_{h}^{*}\left(i-\varsigma_{k h}(i)\right)\right)+\sum_{h=1}^{l} s_{k h} g_{3}\left(\sum_{\vartheta=1}^{\infty} O_{k h}(\vartheta) u_{h}(i-\vartheta)\right)-\sum_{h=1}^{l} s_{k h} g_{3}\left(\sum_{\vartheta=1}^{\infty} O_{k h}(\vartheta) u_{h}^{*}(i-\vartheta)\right)+\left.J_{k}\right|^{2}\right) \\
& \left.-\frac{1}{2}\left|x_{k}\right|^{2}-\frac{1}{2}\left|y_{k}\right|^{2}\right\} \\
& \leq \rho^{i}\left\{\frac{1}{2}\left(\rho\left|a_{k}\right|^{2}-1\right)\left|x_{k}(i)\right|^{2}+\frac{1}{2} \rho \sum_{h=1}^{l}\left|b_{k h}\right|^{2} L_{1}^{2}\left|y_{h}(i)\right|^{2}+\rho \frac{1}{2} \sum_{h=1}^{l}\left|d_{k h}\right|^{2} L_{2}^{2}\left|y_{h}\left(i-\tau_{k h}(i)\right)\right|^{2}+\frac{1}{2} \rho \sum_{h=1}^{l}\left|e_{k h}\right|^{2}\right. \\
& \times L_{3}^{2} \sum_{v=1}^{\infty}\left|M_{k h}(v)\right|^{2}\left|y_{h}(i-v)\right|^{2}+\frac{1}{2} \rho\left|I_{k}\right|^{2}+\frac{1}{2}\left(\rho\left|p_{k}\right|^{2}-1\right)\left|y_{k}(i)\right|^{2}+\frac{1}{2} \rho \sum_{h=1}^{l}\left|q_{k h}\right|^{2} N_{1}^{2}\left|x_{h}(i)\right|^{2}+\frac{1}{2} \rho \\
& \left.\times \sum_{h=1}^{l}\left|r_{k h}\right|^{2} N_{2}\left|x_{h}\left(i-\varsigma_{k h}(i)\right)\right|^{2}+\frac{1}{2} \rho \sum_{h=1}^{l}\left|s_{k h}\right|^{2} N_{3} \sum_{\vartheta=1}^{\infty}\left|O_{k h}(\vartheta)\right|^{2}\left|x_{h}(i-\vartheta)\right|^{2}+\frac{1}{2} \rho\left|J_{k}\right|^{2}\right\} \\
& \leq \rho^{i}\left\{\frac{1}{2} \sum_{h=1}^{l}\left(\rho\left|a_{k}\right|^{2}-1\right)\left|x_{k}(i)\right|^{2}+\frac{1}{2} \sum_{h=1}^{l}\left(\rho\left|p_{k}\right|^{2}-1\right)\left|y_{k}(i)\right|^{2}+\frac{1}{2} \rho \sum_{h=1}^{l}\left|r_{k h}\right|^{2} N_{2}\left|x_{h}\left(i-\zeta_{k h}(i)\right)\right|^{2}+\frac{1}{2} \rho\right. \\
& \times \sum_{h=1}^{l}\left|d_{k h}\right|^{2} L_{2}^{2}\left|y_{h}\left(i-\tau_{k h}(i)\right)\right|^{2}+\frac{1}{2} \rho \sum_{h=1}^{l}\left|e_{k h}\right|^{2} L_{3}^{2} \sum_{v=1}^{\infty}\left|M_{k h}(v)\right|^{2}\left|y_{h}(i-v)\right|^{2}+\frac{1}{2} \rho\left|I_{k}\right|^{2}+\frac{1}{2} \rho \sum_{h=1}^{l}\left|s_{k h}\right|^{2} \\
& \left.\times N_{3} \sum_{\vartheta=1}^{\infty}\left|O_{k h}(\vartheta)\right|^{2}\left|x_{h}(i-\vartheta)\right|^{2}+\frac{1}{2} \rho\left|J_{k}\right|^{2}+\rho\left|q_{k h}\right|^{2} N_{1}^{2}\left|x_{h}(i)\right|^{2}+\rho\left|b_{k h}\right|^{2} L_{1}^{2}\left|y_{h}(i)\right|^{2}\right\} \\
& \Delta v_{k}^{(2)}(i)=v_{k}^{(2)}(i+1)-v_{k}^{(2)}(i) \\
& =\rho^{i}\left\{\frac{1}{2} \rho \sum_{h=1}^{l}\left|d_{k h}\right|^{2} L_{2}^{2} \sum_{\xi=i+1-\tau_{k h}(i+1)}^{i}\left|x_{h}(\xi)\right|^{2}+\frac{1}{2} \rho \sum_{h=1}^{l}\left|r_{k h}\right|^{2} N_{2}^{2} \sum_{\xi=i+1-\varsigma_{k h}(i+1)}^{i}\left|y_{h}(\xi)\right|^{2}\right. \\
& \left.-\frac{1}{2} \sum_{h=1}^{l}\left|d_{k h}\right|^{2} L_{2}^{2} \sum_{\xi=i-\tau_{k h}(i)}^{i-1}\left|x_{h}(\xi)\right|^{2}-\frac{1}{2} \sum_{h=1}^{l}\left|r_{k h}\right|^{2} N_{2}^{2} \sum_{\xi=i-\varsigma_{k h}(i)}^{i-1}\left|y_{h}(\xi)\right|^{2}\right\}
\end{aligned}
$$

Since, $1<\tau_{k h}(i+1)<\tau_{k h}(i)+1$ and $1<\varsigma_{k h}(i+1)<\varsigma_{k h}(i)+1$

$$
\begin{aligned}
\leq & \rho^{i}\left\{\frac{1}{2} \rho \sum_{h=1}^{l}\left|d_{k h}\right|^{2} L_{2}^{2} \sum_{\xi=i-\tau_{k h}(i)}^{i}\left|x_{h}(\xi)\right|^{2}+\frac{1}{2} \rho \sum_{h=1}^{l}\left|r_{k h}\right|^{2} N_{2}^{2} \sum_{\xi=i-\varsigma_{k h}(i)}^{i}\left|y_{h}(\xi)\right|^{2}-\frac{1}{2} \sum_{h=1}^{l}\left|d_{k h}\right|^{2} L_{2}^{2}\right. \\
& \left.\times \sum_{\xi=i-\tau_{k h}(i)}^{i-1}\left|x_{h}(\xi)\right|^{2}-\frac{1}{2} \sum_{h=1}^{l}\left|r_{k h}\right|^{2} N_{2}^{2} \sum_{\xi=i-\varsigma_{k h}(i)}^{i-1}\left|y_{h}(\xi)\right|^{2}\right\} \\
= & \rho^{i}\left\{\frac{1}{2} \rho \sum_{h=1}^{l}\left|d_{k h}\right|^{2} L_{2}^{2}\left|x_{h}(i)\right|^{2}+\frac{1}{2} \rho \sum_{h=1}^{l}\left|d_{k h}\right|^{2} L_{2}^{2} \sum_{\xi=i-\tau_{k h}(i)}^{i-1}\left|x_{h}(\xi)\right|^{2}+\frac{1}{2} \rho \sum_{h=1}^{l}\left|r_{k h}\right|^{2} N_{2}^{2}\left|y_{h}(i)\right|^{2}\right.
\end{aligned}
$$




$$
\begin{aligned}
+ & \frac{1}{2} \rho \sum_{h=1}^{l}\left|r_{k h}\right|^{2} N_{2}^{2} \sum_{\xi=i-\varsigma_{k h}(i)}^{i-1}\left|y_{h}(\xi)\right|^{2}-\frac{1}{2} \sum_{h=1}^{l}\left|d_{k h}\right|^{2} L_{2}^{2} \sum_{\xi=i-\tau_{k h}(i)}^{i-1}\left|x_{h}(\xi)\right|^{2}-\frac{1}{2} \sum_{h=1}^{l}\left|r_{k h}\right|^{2} N_{2}^{2} \\
& \left.\times \sum_{\xi=i-\varsigma_{k h}(i)}^{i-1}\left|y_{h}(\xi)\right|^{2}\right\} \\
\leq & \rho^{i}\left\{\frac{1}{2} \rho \sum_{h=1}^{l}\left|d_{k h}\right|^{2} L_{2}^{2}\left|x_{h}(i)\right|^{2}+\frac{1}{2} \rho \sum_{h=1}^{l}\left|r_{k h}\right|^{2} N_{2}^{2}\left|y_{h}(i)\right|^{2}\right\} \\
v_{3}(i)= & \frac{1}{2} \rho^{i} \sum_{h=1}^{l} e_{k h}^{2} L_{3}^{2} \sum_{v=1}^{\infty} M_{k h}(v) \sum_{r=i-v}^{i-1}\left|x_{k}(r)\right|^{2}+\frac{1}{2} \rho^{i} \sum_{h=1}^{l} s_{k h}^{2} N_{3}^{2} \sum_{\vartheta=1}^{\infty} O_{k h}(\vartheta) \sum_{r=i-\vartheta}^{i-1}\left|y_{k}(r)\right|^{2} \\
\Delta v_{k}^{(3)}(i)= & \rho^{i}\left\{\frac{1}{2} \rho \sum_{h=1}^{l} e_{k h}^{2} L_{3}^{2} \sum_{v=1}^{\infty} M_{k h}(v) \sum_{r=i-v+1}^{i}\left|x_{k}(r)\right|^{2}+\frac{1}{2} \rho \sum_{h=1}^{l} s_{k h}^{2} N_{3}^{2} \sum_{\vartheta=1}^{\infty} O_{k h}(\vartheta) \sum_{r=i-\vartheta+1}^{i}\left|y_{k}(r)\right|^{2}\right. \\
& \left.-\frac{1}{2} \sum_{h=1}^{l} e_{k h}^{2} L_{3}^{2} \sum_{v=1}^{\infty} M_{k h}(v) \sum_{r=i-v}^{i-1}\left|x_{k}(r)\right|^{2}-\frac{1}{2} \sum_{h=1}^{l} s_{k h}^{2} N_{3}^{2} \sum_{\vartheta=1}^{\infty} O_{k h}(\vartheta) \sum_{r=i-\vartheta}^{i-1}\left|y_{k}(r)\right|^{2}\right\}
\end{aligned}
$$

Substitute (17)-(19) this in (16), we obtain,

$$
\begin{aligned}
\Delta v_{k}(i) \leq & \rho^{i}\left\{\frac{1}{2} \sum_{h=1}^{l}\left(\rho\left|a_{k}\right|^{2}-1\right)\left|x_{k}(i)\right|^{2}+\frac{1}{2} \sum_{h=1}^{l}\left(\rho\left|p_{k}\right|^{2}-1\right)\left|y_{k}(i)\right|^{2}+\frac{1}{2} \rho \sum_{h=1}^{l}\left|r_{k h}\right|^{2} N_{2}^{2}\left|x_{h}\left(i-\zeta_{k h}(i)\right)\right|^{2}\right. \\
& +\frac{1}{2} \rho \sum_{h=1}^{l}\left|d_{k h}\right|^{2} L_{2}^{2}\left|y_{h}\left(i-\tau_{k h}(i)\right)\right|^{2}+\frac{1}{2} \rho \sum_{h=1}^{l}\left|e_{k h}\right|^{2} L_{3}^{2} \sum_{v=1}^{\infty}\left|M_{k h}(v)\right|^{2}\left|y_{h}(i-v)\right|^{2}+\frac{1}{2} \rho\left|I_{k}\right|^{2} \\
& +\frac{1}{2} \rho \sum_{h=1}^{l}\left|s_{k h}\right|^{2} N_{3}^{2} \sum_{\vartheta=1}^{\infty}\left|O_{k h}(\vartheta)\right|^{2}\left|x_{h}(i-\vartheta)\right|^{2}+\frac{1}{2} \rho\left|J_{k}\right|^{2}+\frac{1}{2} \rho \sum_{h=1}^{l}\left|d_{k h}\right|^{2} L_{2}^{2}\left|x_{h}(i)\right|^{2} \\
& +\frac{1}{2} \rho \sum_{h=1}^{l}\left|r_{k h}\right|^{2} N_{2}^{2}\left|y_{h}(i)\right|^{2}+\frac{1}{2} \rho \sum_{h=1}^{l} e_{k h}^{2} L_{3}^{2} \sum_{v=1}^{\infty} M_{k h}(v) \sum_{r=i-v+1}^{i}\left|x_{k}(r)\right|^{2} \\
+ & \frac{1}{2} \rho \sum_{h=1}^{l} s_{k h}^{2} N_{3}^{2} \sum_{\vartheta=1}^{\infty} O_{k h}(\vartheta) \sum_{r=i-\vartheta+1}^{i}\left|y_{k}(r)\right|^{2}-\frac{1}{2} \sum_{h=1}^{l} e_{k h}^{2} L_{3}^{2} \sum_{v=1}^{\infty} M_{k h}(v) \sum_{r=i-v}^{i-1}\left|x_{k}(r)\right|^{2} \\
& \left.-\frac{1}{2} \sum_{h=1}^{l} s_{k h}^{2} N_{3}^{2} \sum_{\vartheta=1}^{\infty} O_{k h}(\vartheta) \sum_{r=i-\vartheta}^{i-1}\left|y_{k}(r)\right|^{2}+\rho \sum_{h=1}^{l}\left|q_{k h}\right|^{2} N_{1}^{2}\left|x_{h}(i)\right|^{2}+\rho \sum_{h=1}^{l}\left|b_{k h}\right|^{2} L_{1}^{2}\left|y_{h}(i)\right|^{2}\right\} \\
\leq & \frac{1}{2} \rho^{i}\left\{\sum_{h=1}^{l}\left(\rho\left|a_{k}\right|^{2}+\rho \sum_{h=1}^{l} e_{k h}^{2} L_{3}^{2} \sum_{v=1}^{\infty} M_{k h}(v) \rho \sum_{k=1}^{l}\left|d_{h k}\right|^{2} L_{2}^{2}+\rho \sum_{k=1}^{l}\left|q_{h k}\right|^{2} N_{1}^{2}-1\right)\left|x_{k}(i)\right|^{2}\right. \\
+ & \sum_{h=1}^{l}\left(\rho\left|p_{k}\right|^{2}+\rho \sum_{h=1}^{l} s_{k h}^{2} N_{3}^{2} \sum_{\vartheta=1}^{\infty} O_{k h}(\vartheta) \rho \sum_{k=1}^{l}\left|r_{h k}\right|^{2} N_{2}^{2}+\rho \sum_{k=1}^{l}\left|b_{h k}\right|^{2} L_{1}^{2}-1\right)\left|y_{k}(i)\right|^{2} \\
+ & \rho \sum_{h=1}^{l}\left|r_{k h}\right|^{2} N_{2}^{2}\left|x_{h}\left(i-\zeta_{k h}(i)\right)\right|^{2}+\rho \sum_{h=1}^{l}\left|d_{k h}\right|^{2} L_{2}^{2}\left|y_{h}\left(i-\tau_{k h}(i)\right)\right|^{2}+\rho\left|I_{k}\right|^{2}+\rho\left|J_{k}\right|^{2}
\end{aligned}
$$




$$
\begin{aligned}
& +\left\{\rho \sum_{h=1}^{l}\left|d_{k h}\right|^{2} L_{2}^{2}+\rho \sum_{h=1}^{l}\left|q_{k h}\right|^{2} N_{1}^{2}\right\}\left|x_{h}(i)\right|^{2}-\left\{\rho \sum_{k=1}^{l}\left|d_{h k}\right|^{2} L_{2}^{2}+\rho \sum_{k=1}^{l}\left|q_{h k}\right|^{2} N_{1}^{2}\right\}\left|x_{k}(i)\right|^{2} \\
& \left.+\left\{\rho \sum_{h=1}^{l}\left|r_{k h}\right|^{2} N_{2}^{2}+\rho \sum_{h=1}^{l}\left|b_{k h}\right|^{2} L_{1}^{2}\right\}\left|y_{h}(i)\right|^{2}-\left\{\rho \sum_{k=1}^{l}\left|r_{h k}\right|^{2} N_{2}^{2}+\rho \sum_{k=1}^{l}\left|b_{h k}\right|^{2} L_{1}^{2}\right\}\left|y_{k}(i)\right|^{2}\right\} \\
& \leq \frac{1}{2} \rho^{i}\left\{\left[\rho\left|a_{k}\right|^{2}+\rho \sum_{h=1}^{l} e_{k h}^{2} L_{3}^{2} \sum_{v=1}^{\infty} M_{k h}(v)+\rho \sum_{k=1}^{l}\left|d_{h k}\right|^{2} L_{2}^{2}+\rho \sum_{k=1}^{l}\left|q_{h k}\right|^{2} N_{1}^{2}+\rho\left|p_{k}\right|^{2}\right.\right. \\
& \left.+\rho \sum_{h=1}^{l} s_{k h}^{2} N_{3}^{2} \sum_{\vartheta=1}^{\infty} O_{k h}(\vartheta)+\rho \sum_{k=1}^{l}\left|r_{h k}\right|^{2} N_{2}^{2}+\rho \sum_{k=1}^{l}\left|b_{h k}\right|^{2} L_{1}^{2}-2\right]\left(\left|x_{k}(i)\right|^{2}+\left|y_{k}(i)\right|^{2}\right) \\
& +\rho \sum_{h=1}^{l}\left(\left|r_{k h}\right|^{2} N_{2}^{2}+\left|d_{k h}\right|^{2} L_{2}^{2}\right)\left(\left|x_{h}\left(i-\zeta_{k h}(i)\right)\right|^{2}+\left|y_{h}\left(i-\tau_{k h}(i)\right)\right|^{2}\right) \\
& +\left\{\rho \sum_{h=1}^{l}\left|d_{k h}\right|^{2} L_{2}^{2}+\rho \sum_{h=1}^{l}\left|q_{k h}\right|^{2} N_{1}^{2}\right\}\left|x_{h}(i)\right|^{2}-\left\{\rho \sum_{k=1}^{l}\left|d_{h k}\right|^{2} L_{2}^{2}+\rho \sum_{k=1}^{l}\left|q_{h k}\right|^{2} N_{1}^{2}\right\}\left|x_{k}(i)\right|^{2} \\
& \left.+\left\{\rho \sum_{h=1}^{l}\left|r_{k h}\right|^{2} N_{2}^{2}+\rho \sum_{h=1}^{l}\left|b_{k h}\right|^{2} L_{1}^{2}\right\}\left|y_{h}(i)\right|^{2}-\left\{\rho \sum_{k=1}^{l}\left|r_{h k}\right|^{2} N_{2}^{2}+\rho \sum_{k=1}^{l}\left|b_{h k}\right|^{2} L_{1}^{2}\right\}\left|y_{k}(i)\right|^{2}+\rho\left|I_{k}\right|^{2}+\rho\left|J_{k}\right|^{2}\right\}
\end{aligned}
$$

From, Theorem 1, $\Delta v(i)<0$.

$$
\begin{aligned}
\Delta v(i) & <0 \Rightarrow v(i) \leq v(0) \\
v(i) & \geq \frac{1}{2}\left|x_{k}(i)\right|^{2}+\frac{1}{2}\left|y_{k}(i)\right|^{2}+\frac{1}{2} \sum_{h=1}^{l} d_{k h}^{2} L_{2}^{2}\left|x_{h}(i)\right|^{2}+\frac{1}{2} \sum_{h=1}^{l} r_{k h}^{2} N_{2}^{2}\left|y_{h}(i)\right|^{2}
\end{aligned}
$$

and

$$
\begin{gathered}
v(0) \geq \frac{1}{2}\left|x_{k}(0)\right|^{2}+\frac{1}{2}\left|y_{k}(0)\right|^{2}+\frac{1}{2} \sum_{h=1}^{l} d_{k h}^{2} L_{2}^{2}\left|x_{h}(0)\right|^{2}+\frac{1}{2} \sum_{h=1}^{l} r_{k h}^{2} N_{2}^{2}\left|y_{h}(0)\right|^{2}+\frac{1}{2} \sum_{h=1}^{l} e_{k h}^{2} L_{3}^{2} \sum_{v=1}^{\infty} M_{k h}(v) \\
\times \sum_{\xi=0-\tau_{k h}(0)}^{-1}\left|x_{h}(0)\right|^{2}+\frac{1}{2} \sum_{h=1}^{l} s_{k h}^{2} N_{3}^{2} \sum_{\vartheta=1}^{\infty} O_{k h}(\vartheta) \sum_{\xi=0-\zeta_{k h}(0)}^{-1}\left|y_{h}(0)\right|^{2} \\
v(i) \leq v(0) \leq \frac{1}{2} \sum_{h=1}^{l}\left(e_{k h}^{2} L_{3}^{2}\right) \sum_{-\tau_{k h}}^{0}\left|x_{k}\right|^{2}+\frac{1}{2} \sum_{h=1}^{l}\left(s_{k h}^{2} N_{3}^{2}\right) \sum_{-\zeta_{k h}}^{0}\left|y_{k}\right|^{2} \\
\left|x_{k}(i)\right|^{2}+\left|y_{k}\right|^{2} \leq \frac{1}{2} \sum_{h=1}^{l} \kappa\left(\sup _{\gamma \in(-\tau, 0)}\left|x_{k}(i)\right|^{2}+\sup _{\gamma \in(-\zeta, 0)}\left|y_{k}\right|^{2}\right)
\end{gathered}
$$

Here, $\kappa=\max \left\{e_{k h}^{2} L_{3}^{2}, s_{k h}^{2} N_{3}^{2}\right\}$. Hence, the periodic solution of given system (2) is exponential stable.

\section{Illustrative Example}

In this section, to show the exactness of this proposed work a numerical simulation is presented. In this example, we consider two-dimensional DBAMNNs with discrete and distributed delays of two neurons.

Example 1. The discrete time BAMNNs with mixed time varying delays are considered as follows 


$$
\begin{aligned}
u_{k}(i+1)= & -A u_{k}(i)+\sum_{h=1}^{l} B f_{1}\left(v_{h}(i)\right)+\sum_{h=1}^{l} D f_{2}\left(v_{h}\left(i-\tau_{k h}(i)\right)\right) \\
& +\sum_{h=1}^{l} E f_{3}\left(\sum_{v=1}^{\infty} M(v) v_{h}(i-v)\right)+I_{k} \\
v_{k}(i+1)= & -P v_{k}(i)+\sum_{h=1}^{l} Q g_{1}\left(u_{h}(i)\right)+\sum_{h=1}^{l} R g_{2}\left(u_{h}\left(i-\varsigma_{k h}(i)\right)\right) \\
& +\sum_{h=1}^{l} S g_{3}\left(\sum_{\vartheta=1}^{\infty} O(\vartheta) u_{h}(i-\vartheta)\right)+J_{k}
\end{aligned}
$$

Here,

$$
\begin{gathered}
A=\left(\begin{array}{cc}
0.5 & 0 \\
0 & 0.01
\end{array}\right), B=\left(\begin{array}{cc}
-1.02 & 1.3 \\
0.5 & 0.6
\end{array}\right), D=\left(\begin{array}{cc}
0.03 & 0.3 \\
0.02 & 0.4
\end{array}\right) E=\left(\begin{array}{cc}
1.03 & 0.01 \\
0.5 & 0.6
\end{array}\right) \\
P=\left(\begin{array}{cc}
0.02 & 0 \\
0 & 0.1
\end{array}\right) Q=\left(\begin{array}{cc}
0.03 & 0.5 \\
0.1 & -0.6
\end{array}\right), R=\left(\begin{array}{cc}
-0.5 & 0.3 \\
0.5 & 0.04
\end{array}\right), S=\left(\begin{array}{cc}
0.3 & 0.3 \\
0.2 & 0.4
\end{array}\right)
\end{gathered}
$$

and

$$
\begin{aligned}
f_{1}\left(v_{h}(i)\right) & =-0.1 \sin v_{h}(i) ; f_{2}\left(v_{h}\left(i-\tau_{k h}(i)\right)\right)=-0.8 \sin v_{h}\left(i-\tau_{k h}(i)\right) ; f_{3}\left(v_{h}(i-v)\right)=-0.1 \cos v_{h}(i-v) ; \\
g_{1}\left(u_{h}(i)\right) & =-0.1 \sin u_{h}(i) ; g_{2}\left(u_{h}\left(i-\varsigma_{k h}(i)\right)\right)=0.5 \sin \left(v_{h}\left(i-\varsigma_{k h}(i)\right)\right) ; g_{3}\left(v_{h}(i-\vartheta)\right)=-0.1 e^{u_{h}(i-\vartheta) ;} \\
\tau_{k h}(i) & =\varsigma_{k h}(i)=0.25 i+2 ; M_{k h}=0.05 e^{i} ; O_{k h}=0.5 e^{i} ;
\end{aligned}
$$

From our observations, all the conditions in Theorems 1 and 2 are satisfied for Lipschizt constant 1 and $\rho=1$. Hence the DDBAMNNs (2) has a unique $2 \pi$-periodic solution which is exponentially stable. These facts are also supported by the illustrative numerical simulations, see Figure 1.
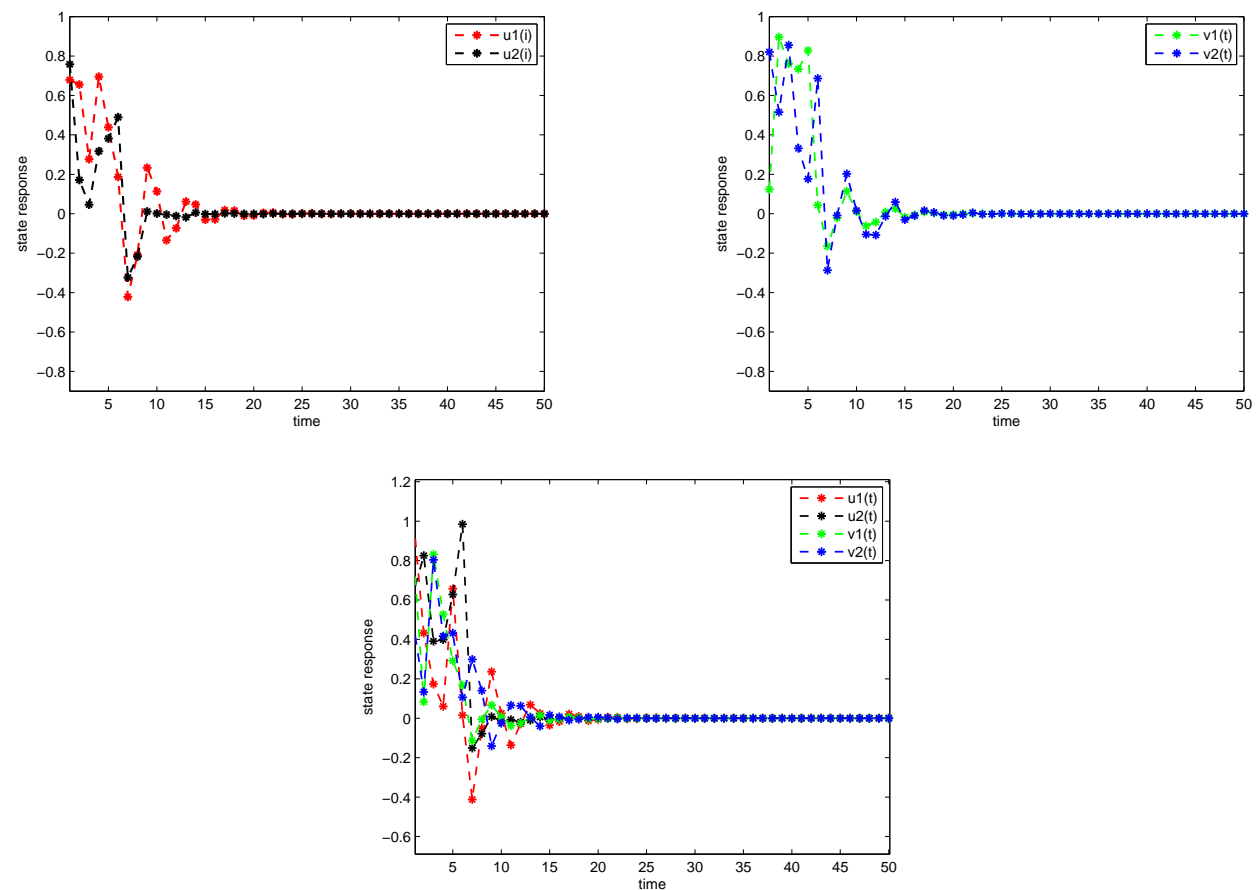

Figure 1. The state response $u(i)$ and $v(i)$ of (20). 


\section{Conclusions}

In this manuscript, we have studied the existence, uniqueness and exponential stability of a periodic solution of discrete time BAMNNs with discrete and infinitely distributed delays. By using the global Lyapunov functional, coincidence degree theory combined with Krichhoff's matrix tree theorem, the sufficient conditions are derived. It should be noted that the method and techniques presented in this manuscript are more precisely variations on existing methods like the LMI approach, the method of the variation of parameters and so on. At the end of this work, we have given two examples to conclude and justify our main results.

Author Contributions: Conceptualization, M.I. and R.R.; methodology, G.R., J.C. and C.H.; validation, J.C. and C.H.; formal analysis, M.I., R.R. and J.C.; investigation, M.I., J.C. and J.A.; writing-original draft preparation, M.I., R.R. and J.C.; writing-review and editing, J.A. and C.H.; supervision, J.C. and C.H.; project administration, J.C. and C.H.; funding acquisition, J.C. and C.H.. These authors contributed equally to this work.

Funding: This work was partially supported by the National Natural Science Foundation of P. R. China (Nos. 11971076, 71861008), RUSA-Phase 2.0 Grant No.F 24-51/2014-U, Policy (TN Multi-Gen), Department of Education Govt. of India, UGC-SAP (DRS-I) Grant No.F.510/8/DRS-I/2016(SAP-I) and DST (FIST - level I) 657876570 Grant No.SR/FIST/MS-I/2018/17.

Conflicts of Interest: The authors declare no conflict of interest.

\section{References}

1. Chen, T.; Huang, L.; Yu, P.; Huang, W. Bifurcation of limit cycles at infinity in piecewise polynomial systems. Nonlinear Anal. Real World Appl. 2018, 41, 82-106. [CrossRef]

2. Huang, C.; Cao, J.; Wen, F.; Yang, X. Stability Analysis of SIR Model with Distributed Delay on Complex Networks. PLoS ONE 2016, 11, e0158813. [CrossRef] [PubMed]

3. Hu, H.; Yi, T.; Zou, X. On spatial-temporal dynamics of Fisher-KPP equation with a shifting environment. Proc. Am. Math. Soc. 2019. [CrossRef]

4. Hu, H.; Yuan, X.; Huang, L.; Huang, C. Global dynamics of an SIRS model with demographics and transfer from infectious to susceptible on heterogeneous networks. Math. Biosci. Eng. 2019, 16, 5729-5749. [CrossRef] [PubMed]

5. Hu, H.; Zou, X. Existence of an extinction wave in the fisher equation with a shifting habitat. Proc. Am. Math. Soc. 2017, 145, 4763-4771. [CrossRef]

6. Huang, C.; Long, X.; Huang, L.; Fu, S. Stability of almost periodic Nicholson's blowflies model involving patch structure and mortality terms. Can. Math. Bull. 2019. [CrossRef]

7. Huang, Y.; Chen, X.; Zhu, H.; Huang, C.; Tian, Z. The Heterogeneous Effects of FDI and Foreign Trade on CO2 Emissions: Evidence from China. Math. Probl. Eng. 2019, 2019, 9612492. [CrossRef]

8. Kumari, S.; Chugh, R.; Cao, J.; Huang, C. Multi Fractals of Generalized Multivalued Iterated Function Systems in b-Metric Spaces with Applications. Mathematics 2019, 7, 967. [CrossRef]

9. Tan, Y.; Huang, C.; Sun, B.; Wang, T. Dynamics of a class of delayed reaction-diffusion systems with Neumann boundary condition. J. Math. Anal. Appl. 2018, 458, 1115-1130. [CrossRef]

10. Tan, Y.; Zhang, M. Global exponential stability of periodic solutions in a nonsmooth model of hematopoiesis with time-varying delays. Math. Methods Appl. Sci. 2017, 40, 5986-5995. [CrossRef]

11. Wang, J.; Huang, C.; Huang, L. Discontinuity-induced limit cycles in a general planar piecewise linear system of saddleCfocus type. Nonlinear Anal. Hybrid Syst. 2019, 33, 162-178. [CrossRef]

12. Yang, X.; Wen, S.; Liu, Z.; Li, C.; Huang, C. Dynamic Properties of Foreign Exchange Complex Network. Mathematics 2019, 7, 832. [CrossRef]

13. Kosko, B. Adaptive bidirectional associative memories. Appl. Opt. 1987, 26, 4947-4960. [CrossRef] [PubMed]

14. Rajchakit, G.; Pratap, A.; Raja, R.; Cao, J.; Alzabut, J.; Huang, C. Hybrid Control Scheme for Projective Lag Synchronization of RiemannCLiouville Sense Fractional Order Memristive BAM Neural Networks with Mixed Delays. Mathematics 2019, 7, 759. [CrossRef]

15. Zuo, Y.; Wang, Y.; Liu, X. Adaptive robust control strategy for rhombus-type lunar exploration wheeled mobile robot using wavelet transform and probabilistic neural network. Comput. Appl. Math. 2018, 37, 314-337. [CrossRef] 
16. Kosko, B. Bidirectional associative memories. IEEE Trans. Syst. Man Cybern. 1988, 18, 49-60. [CrossRef]

17. Zhu, Q.; Huang, C.; Yang, X. Exponential stability for stochastic jumping BAM neural networks with time-varying and distributed delays. Nonlinear Anal. Hybrid Syst. 2011, 5, 52-77. [CrossRef]

18. Yang, C.; Huang, L.; Li, F. Exponential synchronization control of discontinuous nonautonomous networks and autonomous coupled networks. Complexity 2018, 2018, 6164786. [CrossRef]

19. Duan, L.; Huang, L.; Guo, Z.; Fang, X. Periodic attractor for reactiondiffusion high-order hopfield neural networks with time-varying delays. Comput. Math. Appl. 2017, 73, 233-245. [CrossRef]

20. Aouiti, C.; Gharbia, I.B.; Cao, J.; Alsaedi, A. Dynamics of impulsive neutral-type BAM neural networks. J. Frankl. Inst. 2019, 356, 2294-2324.

21. Su, H.; Zhao, Y.; Li, W.; Ding, X. A graph-theoretic approach to exponential stability of stochastic BAM neural networks with time-varying delays. Neural Comput. Appl. 2016, 27, 2055-2063. [CrossRef]

22. Zhang, Z.; Zhou, D. Existence and global exponential stability of a periodic solution for a discrete-time interval general BAM neural networks. J. Frank. Inst. 2010, 347, 763-780. [CrossRef]

23. Cai, Z.; Huang, J.; Huang, L. Generalized Lyapunov-Razumikhin method for retarded differential inclusions: Applications to discontinuous neural networks. Discret. Contin. Dyn. Syst. Ser. B 2017, 22, 3591-3614. [CrossRef]

24. Duan, L.; Fang, X.; Huang, C. Global exponential convergence in a delayed almost periodic nicholsons blowflies model with discontinuous harvesting. Math. Methods Appl. Sci. 2017, 41, 1954-1965. [CrossRef]

25. Huang, C.; Liu, B.; Tian, X.; Yang, L.; Zhang, X. Global convergence on asymptotically almost periodic SICNNs with nonlinear decay functions. Neural Process. Lett. 2019, 49, 625-641. [CrossRef]

26. Agliari, E.; Migliozzi, D.; Tantari, D. Non-convex Multi-species Hopfield Models. J. Stat. Phys. 2018, 172, 1247-1269. [CrossRef]

27. Kurchan, J.; Peliti, L.; Saber, M. Bidirectional associative memories. J. Phys. 1994, 11, 1627-1639. [CrossRef]

28. Liu, X.; Tang, M.; Martin, R.; Liu, X. Discrete-time BAM neural networks with variable delays. Phys. Lett. A 2007, 357, 322-330.

29. Maharajan, C.; Raja, R.; Cao, J.; Rajchakit, G.; Tu, Z.; Alsaedif, A. LMI-based results on exponential stability of BAM-type neural networks with leakage and both time-varying delays: A non-fragile state estimation approach. Appl. Math. Comput. 2018, 326, 33-55. [CrossRef]

30. Maharajana, C.; Raja, R.; Cao, J.; Rajchakit, G. Novel global robust exponential stability criterion for uncertain inertial-type BAM neural networks with discrete and distributed time-varying delays via Lagrange sense. J. Frank. Inst. 2018, 355, 4727-4754. [CrossRef]

31. Sowmiya, C.; Raja, R.; Cao, J.; Li, X. Rajchakit, G. Discrete-time stochastic impulsive BAM neural networks with leakage and mixed time delays: An exponential stability problem. J. Frank. Inst. 2018, 355, 4404-4435.

32. Huang, C.; Liu, B. New studies on dynamic analysis of inertial neural networks involving non-reduced order method. Neurocomputing 2019, 325, 283-287. [CrossRef]

33. Huang, C.; Zhang, H. Periodicity of non-autonomous inertial neural networks involving proportional delays and non-reduced order method. Int. J. Biometh. 2019, 12, 1950016. [CrossRef]

34. Huang, C.; Su, R.; Cao, J.; Xiao, S. Asymptotically stable high-order neutral cellular neural networks with proportional delays and D operators. Math. Comput. Simul. 2019. [CrossRef]

35. Mohamad, S.; Gopalsamy, K. Exponential stability of continuous-time and discrete-time cellular neural networks with delays. Appl. Math. Comput. 2003, 135, 17-38. [CrossRef]

36. Huang, C.; Zhang, H.; Cao, J.; Hu, H. Stability and Hopf bifurcation of a delayed prey-predator model with disease in the predator. Int. J. Bifurc. Chaos 2019, 29, 1950091. [CrossRef]

37. Huang, C.; Zhang, H.; Huang, L. Almost periodicity analysis for a delayed Nicholson's blowflies model with nonlinear density-dependent mortality term. Commun. Pure Appl. Anal. 2019, 18, 3337-3349. [CrossRef]

38. Yue, D.; Li, H. Synchronization stability of continuous/discrete complex dynamical networks with interval time-varying delays. Neurocomputing 2010, 73, 809-891. [CrossRef]

39. Liang, J.; Cao, J. Global asymptotic stability of bi-directional associative memory networks with distributed delays. Appl. Math. Comput. 2004, 152, 415-424. [CrossRef]

40. Huang, C.; Qiao, Y.; Huang, L.; Agarwal, R. Dynamical behaviors of a food-chain model with stage structure and time delays. Adv. Differ. Equ. 2018, 2018, 186. [CrossRef]

41. Huang, C.; Yang, Z.; Yi, T.; Zou, X. On the basins of attraction for a class of delay differential equations with non-monotone bistable nonlinearities. J. Differ. Equ. 2014, 256, 2101-2114. [CrossRef] 
42. Long, X.; Gong, S. New results on stability of Nicholson's blowflies equation with multiple pairs of time-varying delays. Appl. Math. Lett. 2019. [CrossRef]

43. Song, C.; Fei, S.; Cao, J.; Huang, C. Robust Synchronization of Fractional-Order Uncertain Chaotic Systems Based on Output Feedback Sliding Mode Control. Mathematics 2019, 7, 599. [CrossRef]

44. Yang, X.; Zhu, Q.; Huang, C. Generalized lag-synchronization of chaotic mix-delayed systems with uncertain parameters and unknown perturbations. Nonlinear Anal. Real World Appl. 2011, 12, 93-105. [CrossRef]

45. Raja, R.; Sakthivel, R.; Anthoni, S.M. Stability analysis for discrete-time stochastic neural networks with mixed time delays and impulsive effects. Can. J. Phys. 2010, 88, 885-898. [CrossRef]

46. Liu, Y.; Wang, Z.; Liu, X. Robust stability of discrete-time stochastic neural networks with time-varying delays. Neurocomputing 2008, 71, 823-833. [CrossRef]

47. Gao, S.; Li, S.; Wu, B. Periodic solution of discrete time periodic time-vayrying coupled systems on networks. Chaos Solitons Fract. 2017, 103, 246-253. [CrossRef]

48. Huang, C.; Wen, S.; Huang, L. Dynamics of anti-periodic solutions on shunting inhibitory cellular neural networks with multi-proportional delays. Neurocomputing 2019, 357, 47-52. [CrossRef]

49. Wang, J.; Jiang, H.; Hu, C. Existence and stability of periodic solution of discrete-time Cohen-Grossberg neural networks with delays and impulses. Neurocomputing 2014, 142, 542-550. [CrossRef]

50. Zhang, X.; Li, W.; Wang, K. The existence, uniqueness and global exponential stability of periodic solution for a coupled system on networks with time delays. Neurocomputing 2015, 173, 971-978. [CrossRef]

51. Huang, C. Convergence on non-autonomous inertial neural networks with unbounded distributed delays. J. Exp. Theor. Artif. Intell. 2019. [CrossRef]

52. Huang, C.; Cao, J.; Wang, P. Attractor and Boundedness of Switched Stochastic Cohen-Grossberg Neural Networks. Discret. Dyn. Nat. Soc. 2016, 2016, 4958217. [CrossRef]

53. Li, M.Y.; Shuai, Z. Global-stability problem for coupled systems of differential equations on networks. J. Differ. Equ. 2010, 248, 1-20. [CrossRef]

54. Zhang, C.; Li, W.; Wang, K. Boundedness for network of stochastic coupled van der Pol oscillators with time-varying delayed coupling. Appl. Math. Model. 2013, 37, 5394-5402. [CrossRef]

55. Zhang, C.; Li, W.; Su, H.; Wang, K. Asymptotic boundedness for stochastic coupled systems on networks with Markovian switching. Neurocomputing 2014, 136, 180-189. [CrossRef]

56. Zhang, C.; Li, W.; Wang, K. A graph-theoretic approach to stability of neutral stochastic coupled oscillators network with time-varying delayed coupling. Math. Meth. Appl. Sci. 2014, 79, 1179-1190. [CrossRef]

57. Gui, Z.; Ge, W. Existence and uniqueness of periodic solutions of nonautonomous cellular neural networks with impulses. Phys. Lett. A 2006, 354, 84-94. [CrossRef]

58. Xiang, H.; Yan, K.; Wang, B. Existence and global exponential stability of periodic solution for delayed high-order Hopfield-type neural networks. Phys. Lett. A 2006, 352, 341-349. [CrossRef]

59. West, D.B. Introduction to Graph Theory; Prentice Hall: Upper Saller River, NJ, USA, 1996; pp. 1-57.

60. Gaines, R.; Mawhin, J. Coincidence Degree and Nonlinear Diffential Equations; Springer: Berlin, Germany, 1977. 